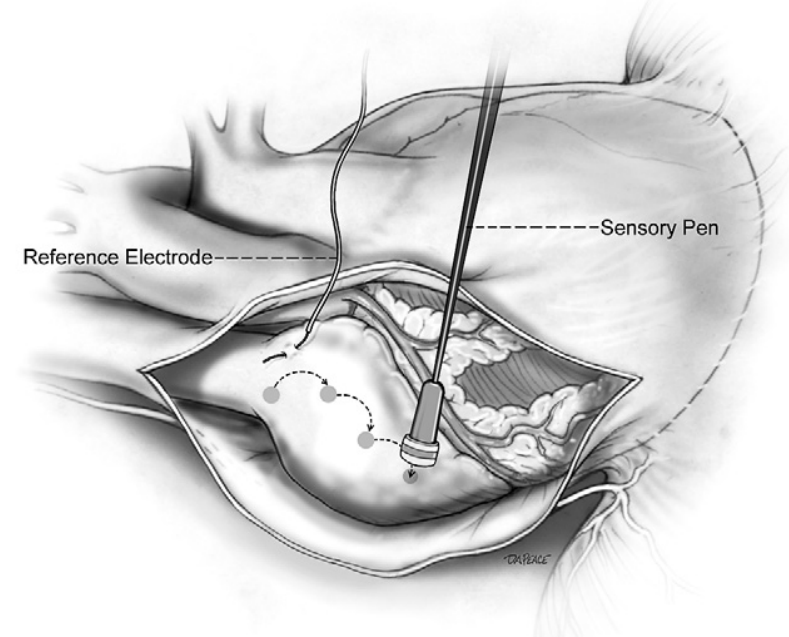

FIGURE 2. Thoracoscopic sensing, pacing, and ablation electrode used to map a migrating focus of tachycardia; the focus migrates, requiring repeat ablations.

\section{DISCUSSION}

Inappropriate sinus tachycardia is challenging for the electrophysiology community because the ablation targets are often located proximal to the phrenic nerve, occasionally resulting in phrenic nerve injury. ${ }^{3-5}$ Video-assisted ablation is a safe alternative that preserves the phrenic nerve. ${ }^{1,2}$

Persistence of inappropriate sinus tachycardia secondary to migration of the earliest site of activation is known to electrophysiologists; however, it may not be known to cardiothoracic surgeons. ${ }^{5}$ Younger surgeons also may not be familiar with activation mapping, which uses a roving sens- ing electrode to compare electrograms from various sites on the atrium with the body surface electrocardiogram $\mathrm{P}$ wave (lead II) and a reference electrode (the wire sutured to the right atrial appendage). The thoracoscopic bipolar pen showed that the earliest site of activation was migrating down the atrium, although it was eventually ablated. Man and colleagues ${ }^{4}$ reported 13 of 22 patients in the catheterization laboratory had a cranial to caudal migration of their activation sites with an eventual abrupt change in P-wave morphology. Marrouche and colleagues ${ }^{5}$ used a 3-dimensional mapping system in the catheterization suite and found that that the inciting focus of tachycardia shifted caudally an average of $23 \pm 11 \mathrm{~mm}$ after ablation. ${ }^{5}$

\section{CONCLUSIONS}

This case describes a migrating focus of inappropriate sinus tachycardia and demonstrates that it can be mapped and ablated by surgeons epicardially with the use of a thoracoscopic pen electrode.

\section{References}

1. Taketani T, Wolf RK, Garrett JV. Partial cardiac denervation and sinus node modification for inappropriate sinus tachycardia. Ann Thorac Surg. 2007;84:652-4.

2. Kreisel D, Bailey M, Lindsay BD, Damiano RJ Jr. A minimally invasive surgical treatment for inappropriate sinus tachycardia. J Thorac Cardiovasc Surg. 2005; 130:598-9.

3. Vatasescu R, Shalganov T, Kardos A, Jalabadze K, Paprika D, Gyorgy M, et al Right diaphragmatic paralysis following endocardial cryothermal ablation of inappropriate sinus tachycardia. Europace. 2006;8:904-6.

4. Man KC, Knight B, Tse HF, Pelosi F, Michaud GF, Flemming M, et al. Radiofrequency catheter ablation of inappropriate sinus tachycardia guided by activation mapping. J Am Coll Cardiol. 2000;35:451-7.

5. Marrouche NF, Beheiry S, Tomassoni G, Cole C, Bash D, Dresing T, et al. Threedimensional nonfluoroscopic mapping and ablation of inappropriate sinus tachycardia. Procedural strategies and long-term outcome. J Am Coll Cardiol. 2002; 39:1046-54.

\title{
Left atrioesophageal fistula following catheter ablation for atrial fibrillation: Off-bypass, primary repair using an extrapericardial approach
}

\author{
Sandeep Khandhar, MD, Stephanie Nitzschke, MD, and Niv Ad, MD, Falls Church, Va
}

\footnotetext{
From the Inova Heart and Vascular Institute, Falls Church, Va.

Disclosures: None.

Received for publication Dec 17, 2008; accepted for publication Dec 27, 2008; available ahead of print March 18, 2009.

Address for reprints: Niv Ad, MD, Inova Heart and Vascular Institute, 3300 Gallows

Rd, Falls Church, VA 22042 (E-mail: nivadmd@hotmail.com).

J Thorac Cardiovasc Surg 2010;139:507-9

$0022-5223 / \$ 36.00$

Copyright (c) 2010 by The American Association for Thoracic Surgery

doi:10.1016/j.jtcvs.2008.12.036
}

Catheter ablation for the treatment of atrial fibrillation is becoming the treatment of choice for drug-refractory symptomatic atrial fibrillation. This technique has increased in popularity, and the number of cases performed each year has risen dramatically. ${ }^{1}$ Although widely utilized, the technique of catheter ablation varies widely among electrophysiology labs. ${ }^{1}$ Overall, this procedure is considered safe, with the incidence of significant complications at $6 \% .^{1}$ This 
report deals with the clinical details and the surgical approaches to left atrioesophageal fistula, a complication with an estimated, but likely underrepresented, incidence of $0.05 \%$. It is of major concern, as the diagnosis can be subtle and often unrecognized, and it can result in a very high mortality rate as documented by the majority of case reports. ${ }^{2}$

We present a case of a left atrioesophageal fistula that was repaired primarily using an extrapericardial approach.

\section{CLINICAL SUMMARY}

A 46-year-old man had catheter-based ablation using radiofrequency energy for persistent atrial fibrillation. Twenty-seven days following the procedure, he was admitted with high fever and found to have streptococcal bacteremia. Cultures revealed multiple Streptococcus spp. The diagnosis of atrioesophageal fistula was entertained but a computed tomography (CT) scan of the chest was initially read as normal. A transthoracic echocardiogram revealed no vegetations, and the patient remained in atrial fibrillation. Treatment with intravenous antibiotics was begun and the patient was observed for several days. A repeat CT scan of the chest and transthoracic echocardiogram were read as unremarkable, and the patient was discharged on postprocedure day 34. Two days later, he developed a transient left hemiparesis and expressive aphasia and was admitted to our institution. An initial CT of the head was negative, and a CT scan of the chest was obtained, revealing dependent air in the region of the left atrium adjacent to the esophagus (Figure 1, $A$ and $B)$. An emergency operation was planned.

\section{OPERATIVE APPROACH}

A left posterolateral thoracotomy was performed through the fifth intercostal space with the left femoral vessels prepped into the field for potential femoral cannulation. An intercostal muscle flap was pedicled posteriorly, and the esophagus was mobilized from the hiatus cephalad to the level of the inferior pulmonary vein. The pericardium was opened longitudinally posterior to the phrenic nerve, exposing the anterior aspect of the superior and inferior left pulmonary veins as they joined the left atrium. Except for an apparent process involving the back of the left atrium, no other significant pathologic findings were identified from within the pericardium. Posterior dissection then ensued in an extrapericardial plane, and pericardial sutures were used to rotate the heart anteromedially, thus exposing the posterior aspects of the pericardial sac. The esophagus was densely adhered to the midportion of the posterior left atrium. The esophagus was separated from the posterior pericardial reflection superiorly and under the aortic arch; this dissection carried caudally just beyond the superior pulmonary vein. Medially, the contralateral pulmonary veins were identified and the fistulous communication clearly isolated at the midportion of the left atrium. From outside the pericardium, a 4-0 polypropylene purse-string suture was placed around the fistula over the posterior aspect of the left atrium, deep enough to include the left atrial wall. The small ( $\sim 1 \mathrm{~cm}$ in diameter) atrioesophageal fistula was then divided with a single fire of the Endo-GIA Universal stapler (Covidien, Norwalk, Conn) (gray, vascular load). The pursestring suture was tied down, and the esophageal aspect of the fistula was oversewn. The intercostal muscle flap was interposed between the left atrium and esophagus and secured to the pericardium. The pericardial defect was closed and the thoracotomy incision closed over a 28-French right-angled chest tube placed near the fistula site.

The patient's postoperative course was uneventful. A barium swallow evaluation was negative on postoperative day 4. His diet was advanced and he was discharged home on postoperative day 7. One-month follow-up revealed no complications; the patient tolerated a regular diet and showed complete neurologic recovery.

\section{DISCUSSION}

The optimal management of isolated atrial fibrillation remains controversial. It is clear that catheter-based techniques are becoming more prevalent. ${ }^{1}$ Collateral damage from the energy source, especially radiofrequency, is a well-identified complication, and associated injury to the esophagus has been well documented. ${ }^{3,4-6}$ These injuries were first recognized in patients having operative radiofrequency ablation using monopolar devices and are now being rediscovered with catheter-based interventions. Despite the precautions taken during catheter ablation, atrioesophageal fistulization occurred, underscoring the lack of full understanding with regard to effective prevention of this complication.

Atrioesophageal fistulas described in earlier surgical reports typically were noted to present in the first 2 weeks after an ablative procedure; however, delay in presentation seems to be somewhat typical in recently published reports. ${ }^{2-4,6,7}$ Presenting symptoms include massive exansanginuation, endocarditis, sepsis, fever, malaise, or neurologic changes. ${ }^{2-5,7}$ Preis and colleagues highlighted the importance of the clinician and radiologist and including this diagnosis in the differential when ordering imaging studies. ${ }^{2}$ A CT of the chest typically shows air within or adjacent to the left atrium, and a head CT can show evidence of embolic infarcts. Instrumentation of the esophagus is contraindicated to prevent enlargement of the fistula tract and air embolization and to avoid further damage to the esophagus.

In our patient, the initial presentation of the atrioesophageal fistula occurred on postprocedure day 27 , and the patient's first neurologic deficit occurred on postprocedure day 36. The diagnosis was based on the clinical presentation and visualization of air behind the left atrium as demonstrated by a chest CT (Figure 1, $A$ and $B$ ). The patient consented for emergency surgery, but the best surgical approach was unclear. A review of the literature on atrioesophageal fistulas 

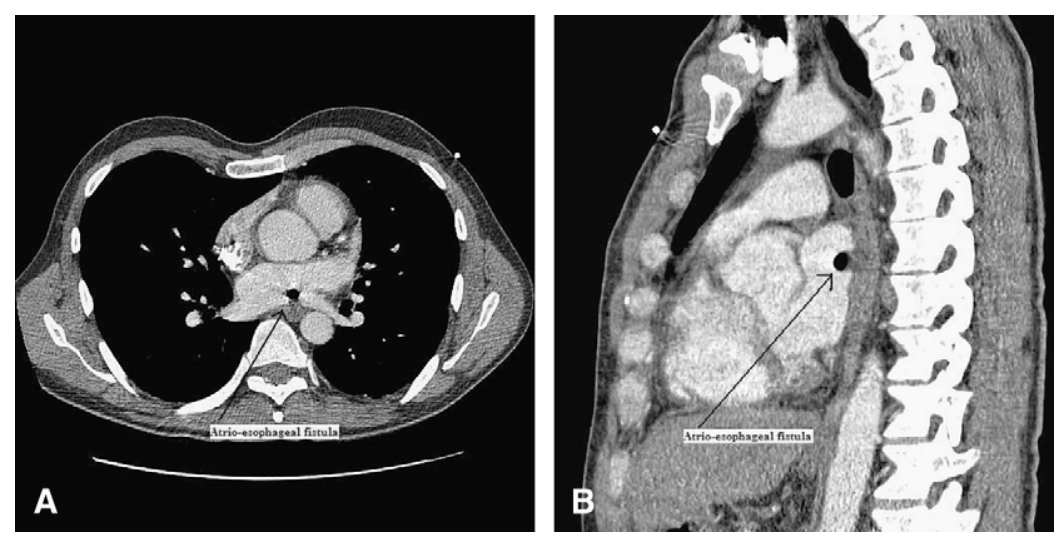

FIGURE 1. A and B, Contrast computed tomography demonstrating air contrast within the cavity of the left atrium.

discusses the difficulty in diagnosis, but there is little discussion on the options for management.

Management options previously described have included esophageal diversion, stenting, and direct operative repair, either intracardiac or thoracic. ${ }^{6,8,9}$ For this patient, we chose an approach that allowed for maximal operative flexibility: left thoracotomy for extracardiac and extrapericardial exposure, femoral access for cardiopulmonary bypass and preparation for cervical esophageal diversion. Our patient was managed successfully via left thoracotomy and resection of the fistula using an extrapericardial approach without the need for cardiopulmonary bypass or esophageal diversion. We find that this approach has several advantages: (1) esophageal stenting requires esophagoscopy, further manipulation of the esophagus, and air insufflation, all of which increase the risk of air embolization; (2) esophageal diversion effectively diminishes the risk of air embolization but does little to prevent ongoing bacterial contamination, especially if a localized abscess cavity is present; (3) intracardiac repair of the fistula requires cardiopulmonary bypass, necessitating heparinization and increasing the risk for cerebral hemorrhage, especially in the setting of recent cerebral embolization and stroke.

Despite previous abandonment of these procedures by some groups, ${ }^{7}$ it has become clear that catheter-based ablation for atrial fibrillation has clinical benefits and there- fore will be increasingly performed as catheter-based techniques continue to evolve. Until technical advances progress to the level of prevention of this problem, early recognition and prompt surgical intervention remain the key factors to successful management of this often devastating complication.

\section{References}

1. Cappato R, Calkins H, Chen SA, Davies W, Iesaka Y, Kalman J, et al. Worldwide survey on the methods, efficacy, and safety of catheter ablation for atrial fibrillation. Circulation. 2005;111:1100-5.

2. Preis O, Digumarthy SR, Wright CD, Shepard JO. Atrioesophageal fistula after catheter pulmonary venous ablation for atrial fibrillation: imaging features. J Thorac Imaging. 2007;22:283-5.

3. Pappone C, Oral H, Santinelli V. Atrio-esophageal fistula as a complication of percutaneous transcatheter ablation of atrial fibrillation. Circulation. 2004; 109:2724-6.

4. Scanavacca M, D'Avila A, Parga J, Sosa E. Left atrial-esophageal fistula following radiofrequency catheter ablation of atrial fibrillation. J Cardiovasc Electrophysiol. 2004; $15: 960-2$

5. Gillinov M, Pettersson G, Rice T. Esophageal injury during radiofrequency ablation for atrial fibrillation. $J$ Thorac Cardiovasc Surg. 2001;122:1239-40.

6. Cummings J, Schweikert R, Saliba W, et al. Brief communication: atrial-esophageal fistulas after radiofrequency ablation. Ann Int Med. 2006;144:572-4.

7. Doll N, Borger M, Fabricius A, et al. Esophageal perforation during left atrial radiofrequency ablation: is the risk too high? J Thorac Cardiovasc Surg. 2003;125: 836-42.

8. Bunch T, Nelson J, Foley T, et al. Temporary esophageal stenting allows healing of esophageal perforations following atrial fibrillation ablation procedures. $J$ Cardiovasc Electrophysiol. 2006;17:435-9.

9. Sonmez B, Demirsoy E, Yagan N, et al. A fatal complication due to radiofrequency ablation for atrial fibrillation: atrio-esophageal fistula. Ann Thorac Surg. 2003;76: 281-3. 